\title{
Wideband analytical equivalent circuit for coupled asymmetrical nonaligned slit arrays
}

\author{
Carlos Molero, ${ }^{*}$ Raúl Rodríguez-Berral ${ }^{\dagger}$ and Francisco Mesa $^{\ddagger}$ \\ Department of Applied Physics 1, Universidad de Sevilla, 41012 Seville, Spain \\ Francisco Medina ${ }^{\S}$ \\ Department of Electronics and Electromagnetism, Universidad de Sevilla, 41012 Seville, Spain
}

(Received 26 September 2016; published 1 February 2017)

\begin{abstract}
Microstructured metallic devices have been extensively studied because of their interesting properties for controlling the transmission, reflection, and absorption of electromagnetic waves. A very simple implementation is an array of infinitely long parallel metal strips printed on a dielectric substrate. In the past few years, several analytical models have been reported based on the use of equivalent circuits with distributed and lumped components to account for the electrical performance of these structures. However, the proposed models are restricted to highly symmetrical configurations of the basic unit cell of the periodic structure. The purpose of this paper is to present the nontrivial extension of such circuit models to deal with nonsymmetrical structures. More specifically, a wideband equivalent-circuit model will be developed to describe the scattering properties of a pair of coupled different nonaligned slit gratings printed on a dielectric slab of arbitrary thickness. The relevant consequences of the lack of symmetry of the structures under study will be thoroughly discussed. The obtained equivalent network can be straightforwardly used to model stacked structures with an arbitrary number of nonsymmetrical striplike arrays.
\end{abstract}

DOI: 10.1103/PhysRevE.95.023303

\section{INTRODUCTION}

The study of the optical scattering properties of periodic metallo-dielectric structures has been a topic of interest for many decades, from the pioneering works of Wood and Rayleigh [1-3] to the most recent findings on extraordinary transmission phenomena [4-7]. This interest has not been restricted to the optical frequency range, with other examples of research found in the infrared [8,9], terahertz [10], and microwave [11-13] domains. The importance of structures of that kind lies in their ability to tailor the reflected, transmitted, or diffracted waves when a plane wave impinges on them. A subclass of electromagnetic periodic structures of particular interest consists of two-dimensional (2D) or 1D distributions of metal patches (2D) or strips (1D) printed on (or embedded in) a layered dielectric medium. The quasicomplementary counterparts made of distributions of planar apertures or slits in thin metal layers are also widely used. The spectral features of these structures make them especially suitable for the design of frequency selective surfaces $[14,15]$, polarizers [16,17], and high-impedance surfaces $[18,19]$.

The analysis of the electrical response of periodic electromagnetic structures requires the solution of Maxwell's equations subject to the appropriate boundary conditions in a single unit cell of the structure. In general, the boundaries that define the unit cell in the numerical or analytical solution of the problem are periodic boundary conditions (PBCs) [20], which can become perfect electric or magnetic conductor virtual boundaries for normal incidence and highly symmetrical scatterers. This fact immediately suggests that the scattering properties of the infinite periodic electromagnetic structure

\footnotetext{
*cmolero@us.es

${ }^{\dagger}$ rrberral@us.es

${ }^{\ddagger}$ mesa@us.es

§medina@us.es
}

can be formulated as a discontinuity waveguide problem, as it has been done, for instance, to analyze extraordinary optical transmission through periodic arrays of electrically small apertures in a metal slab [21-25]. This kind of problem is very familiar to microwave practitioners and has been the subject of intense study for many years [26]. Posing the problem in this manner automatically leads, if desired, to obtaining approximate analytical or quasianalytical models in terms of equivalent circuits. Some recent examples extracted from the literature on 1D thick metal gratings can be found in [27-29]. In comparison with purely numerical approaches, an equivalent circuit is certainly a very useful tool to speed up calculations. Moreover, these circuit models are usually much simpler than highly accurate and elegant analytical solutions available for some practical geometries (for instance, 1D metal or dielectric strip gratings [30-32]). An additional key feature of the equivalent circuits is that they provide good physical insight into the behavior of the periodic device. This is especially true if the circuit parameters can easily be related to the geometric and material parameters of the physical structure. As this feature is very helpful for the design of devices based on this kind of system [33,34], great effort has been made to develop accurate equivalent circuits for planar periodic metallic structures; see, for instance, $[14,35,36]$ and references therein. The authors have also elaborated dynamic equivalent circuits for $1 \mathrm{D}$ arrays of metal strips [37,38] or $2 \mathrm{D}$ arrays of metal patches or apertures [39-41].

Most of the reported equivalent circuits consider a single metalized layer (with a printed periodic pattern) embedded in a layered dielectric environment. However, in many structures of interest, these periodic gratings or grids are stacked to add flexibility to the design process and obtain specific spectral responses not achievable with a single grating. For instance, artificial dielectric media based on stacked arrays of metal strips were proposed in the 1950s [12,13,42,43] and much more recent studies of its $2 \mathrm{D}$ version are reported in $[44,45]$. 
The simplest way of addressing the characterization of stacked planar structures is to consider each grating individually and then use the so-called one-mode interaction approach [46]. Under this approximation, the operation frequency is assumed to be below the diffraction regime threshold and the reactive coupling with adjacent gratings is neglected (only the main beam is considered in the interaction between successive gratings). In the circuit model associated with this simplified approach, the equivalent admittances characterizing the planar grids or gratings are simply connected through a section of transmission line. Within its validity range, this approach is often employed (implicitly or explicitly) with reasonable accuracy [47-49]. However, in many practical cases of interest, the periodically structured surfaces are placed so close to each other as to make the above approximation rather useless (fishnet structures [50,51] are good examples of those situations). Even though the separation between gratings is relatively large and no interaction through evanescent modes or harmonics takes place, the one-mode interaction model should also be dismissed if a high-order harmonic (or mode) is excited above a cutoff in some of the dielectric layers separating the metal gratings. In these cases, more sophisticated methods have been proposed, such as the mutual impedance approach $[52,53]$ or the generalized scattering-matrix method [54]. Although these methods are efficient numerical tools, each individual scattering matrix has still to be obtained using, typically, a method-of-moments formulation [55] or an iterative algorithm [56]. Unfortunately, these numerical methods demand a significant amount of computational resources and do not provide too much physical insight into the behavior of the analyzed structure. Again, the development of analytical equivalent circuits also for this kind of stacked structure not amenable to the one-mode interaction model would be highly desirable.

Very recently, the present authors have proposed a circuit model methodology for stacked 1D strip gratings [57] that is valid for any separation between gratings as well as any value of the slab dielectric constants. The model incorporates the interaction between successive gratings through modes (or Floquet harmonics) of any order. The procedure yields an equivalent $\Pi$ circuit for every pair of adjacent striplike gratings, whose elements are analytically known. This equivalent $\Pi$ circuit has some interesting properties that can advantageously be used to deal with an arbitrary number of stacked gratings. However, the developed circuit model has an important limitation since its derivation was based on the calculation of the equivalent admittances for the considered grating in the presence of a perfect electric-magnetic wall (using the procedure reported in [38]). Thus, the circuit is only valid for gratings having exactly the same geometry, namely, identical distributions of slits with the same width and perfectly aligned (see Fig. 1 in [57]). The purpose of the present paper is then to remove this limitation and develop a circuit model for a pair of coupled striplike gratings having the same period but different slit widths and/or misaligned slits. This problem poses an interesting challenge in the field since a solution in the form of an equivalent circuit is lacking in the literature. Once the circuit model for a single pair of nonsymmetrical striplike gratings has been obtained, the methodology in [57] can be used to deal with stacked structures with an arbitrary number

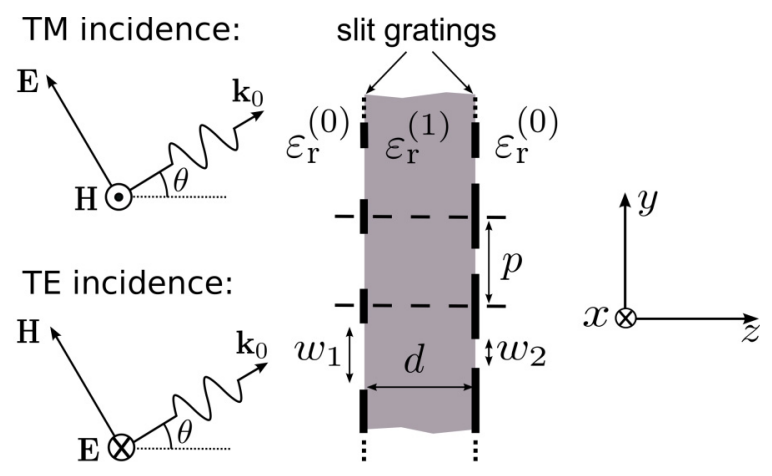

FIG. 1. Cross section of a pair of periodic coupled slit arrays. The metal strips are infinitely thin and infinitely long along the $x$ direction. The slit widths of each grating are, in general, different. $\mathrm{TM}$ or TE oblique incidence is considered.

of gratings. Thus, the following sections present a detailed explanation of the procedure followed to obtain analytical equivalent circuits for a misaligned pair of coupled gratings. The accuracy of the results computed with the proposed model will be systematically checked by comparison with those obtained using commercial electromagnetic solvers (HFSS [58]) as well as the experimental data provided in [59]. Finally, an example of a stacked structure with three gratings is also studied.

\section{DERIVATION OF THE EQUIVALENT П NETWORK}

As stated previously, the equivalent $\Pi$-circuit topology derived in [57] for a pair of identical and aligned coupled slit gratings separated by a dielectric slab of arbitrary thickness and permittivity exhibits excellent performance over a very wide frequency range, even well beyond the onset of the grating-lobe regime. Due to restrictions of the methodology employed in the derivation of that $\Pi$ circuit [57], the unit cell of the structure under study needs to have a vertical symmetry plane (see Fig. 1 in [57]). However, nonsymmetrical stacks of different slit arrays with the same period but different slit widths and relative slit locations have been shown to exhibit interesting properties [59] and, in any case, introduce more flexibility in the design of devices based on those structures. Fortunately, there exists an alternative procedure to obtain a $\Pi$ topology for different coupled slit arrays with the same period, which is based on the matrix formalism of the microwave network analysis [60].

\section{A. Equivalent ח-circuit topology for two different aligned coupled gratings}

The basic structure under study is schematically shown in Fig. 1. A pair of 1D aligned periodic arrays with different slit widths ( $w_{1}$ and $w_{2}$ ), having the same lattice parameter (period $p$ ), are sandwiching a dielectric slab with permittivity $\varepsilon_{r}^{(1)}$ and thickness $d$. (Two slit gratings are considered aligned when the center of their respective slit apertures is located at the same height.) The lack of a vertical symmetry plane perpendicular to the longitudinal $z$ direction clearly precludes the use of the even-odd excitation analysis employed in [57] to derive the corresponding equivalent $\Pi$ circuit. A different strategy 
should then be found to obtain an appropriate $\Pi$ network to model the scattering of a TE- or TM-polarized plane wave that impinges obliquely on the structure, as shown in Fig. 1. Before dealing with the coupled-grating case, it will first be considered the multimode equivalent network for a single slit grating surrounded by two semi-infinite dielectric media. This case was already studied in [38]. The corresponding expression for the equivalent admittance of the aperture discontinuity, given in Eq. (11) of [38], is

$$
Y_{\mathrm{eq}}=\sum_{\forall n \neq 0}^{\infty}\left[\frac{\tilde{f}_{n}(w)}{\tilde{f}_{0}(w)}\right]^{2}\left(Y_{n}^{(0)}+Y_{n}^{(1)}\right),
$$

where $Y_{n}^{(i)}$ is the admittance of the $n$th Floquet harmonic in medium $i=0,1$, defined as

$$
Y_{n}^{(i)}= \begin{cases}\varepsilon_{r}^{(i)} \varepsilon_{0} \omega / \beta_{n}^{(i)} & \text { for TM incidence } \\ \beta_{n}^{(i)} /\left(\mu_{0} \omega\right) & \text { for TE incidence }\end{cases}
$$

with $\varepsilon_{r}^{(i)}$ being the relative permittivity in medium $(i), \varepsilon_{0}$ the free-space permittivity, $\omega$ the angular frequency, and $\beta_{n}^{(i)}$ the wave number of the $n$th harmonic in medium $(i)$, given by

$$
\beta_{n}^{(i)}=\sqrt{\varepsilon_{r}^{(i)} k_{0}^{2}-\left(k_{n}+k_{t}\right)^{2}} .
$$

The parameter $k_{n}=2 n \pi / p$ in (3) stands for the cutoff wave number of the $n$th harmonic, $k_{t}=\sqrt{ } \varepsilon_{r}^{(0)} k_{0} \sin (\theta)$ is the tangential (to the grating) component of the wave vector of the incident plane wave, and $k_{0}$ is the wave number in vacuum. The term $\tilde{f}_{n}(w) / \tilde{f}_{0}(w)$ in $(1)$ represents the ratio between the $n$th and zeroth-order spectral components of the aperture field $\left[\tilde{f}_{n}(w)\right.$ is the Fourier transform of the spatial profile of the tangential electric field at the slit-aperture plane, evaluated at $k=k_{n}+k_{t}$ ]. Following the proposal in [38] for a physically suitable approximation of the slit-aperture field, this spectral ratio can be expressed as

$$
\frac{\tilde{f}_{n}(w)}{\tilde{f}_{0}(w)}= \begin{cases}\frac{J_{0}\left(\left[k_{n}+k_{t}\right] w / 2\right)}{J_{0}\left(k_{t} w / 2\right)} & \text { for TM incidence } \\ \frac{J_{1}\left(\left[k_{n}+k_{t}\right] w / 2\right)}{J_{1}\left(k_{t} w / 2\right)} \frac{k_{t}}{k_{n}+k_{t}} & \text { for TE incidence }\end{cases}
$$

with $w$ being the slit width and $J_{q}(\cdot)$ the $q$ th-order Bessel function of first kind, $q=0,1$. From a circuit standpoint, the expression in (1) can be interpreted as the parallel connection of an infinite number of admittances. Each admittance, recognized as a single addend in the series in (1), is in turn a parallel connection of two semi-infinite $n$th transmission lines with characteristic admittances $Y_{n}^{(0)}$ and $Y_{n}^{(1)}$. Such lines are connected through a transformer with turns ratio $N_{n}=\tilde{f}_{n}(w) / \tilde{f}_{0}(w)$. A schematic representation of the entire network is depicted in Fig. 2.

Let us now consider the coupled-grating system shown in Fig. 1 (the distance between gratings is denoted by $d$ ). Regarding each individual grating separately, their respective circuit models, depicted in Fig. 3(a), have the same representation as the one described in Fig. 2. The information about the slit

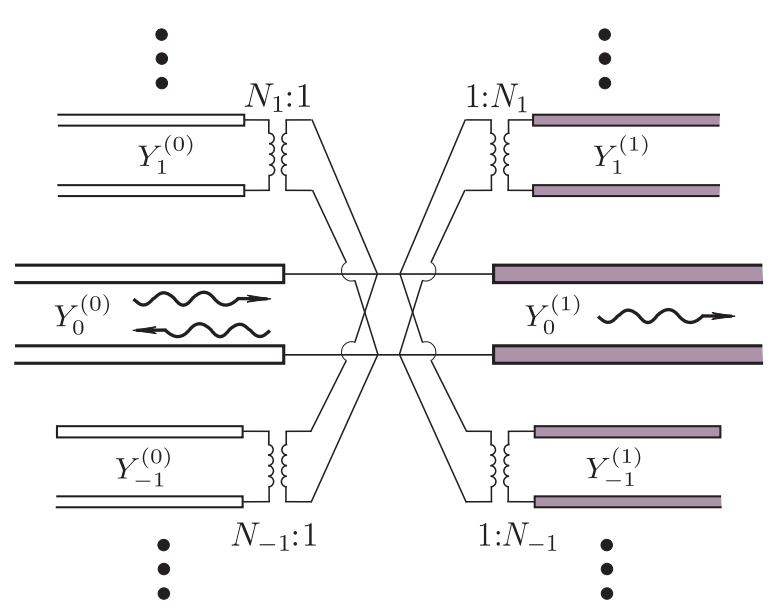

FIG. 2. Multimode equivalent circuit for a single grating placed between two semi-infinite half spaces.

width is included in the transformer ratio expression associated with the $n$th harmonic, which is here defined as

$$
N_{n}\left(w_{i}\right)=\frac{\tilde{f}_{n}\left(w_{i}\right)}{\tilde{f}_{0}\left(w_{i}\right)}
$$

The transformer ratios will be different when $w_{1} \neq w_{2}$. On the contrary, the characteristic admittances and the propagation constants of the $n$th transmission lines in a particular medium are identical to each other regardless of the geometry of the
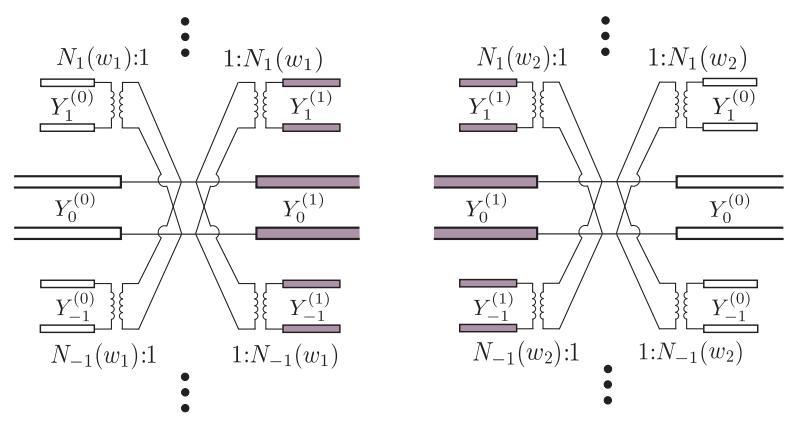

(a)

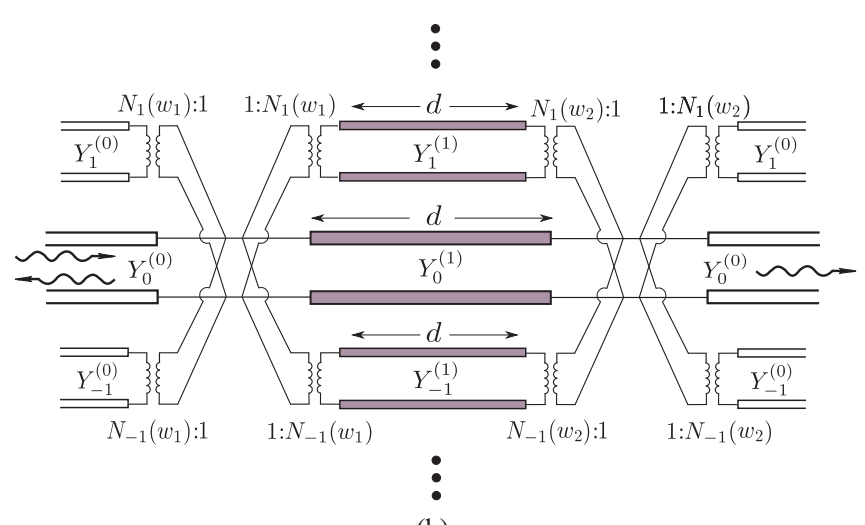

(b)

FIG. 3. (a) Individual equivalent circuits for the slit arrays forming the coupled system. (b) Equivalent circuit of the coupled system, obtained after connecting the $n$th transmission lines of each individual circuit. 
gratings under consideration (they basically depend on the periodicity of the structure and the permittivity of the medium). This fact allows us to construct the multimode circuit for the pair of coupled gratings by simply connecting the transmission lines associated with harmonics of the same order within medium (1), as shown in Fig. 3(b). The length of each $n$th internal line (gray lines in the figure) is precisely the dielectric thickness $d$. In principle, the internal circuit comprises an infinite number of internal lines. However, in practice, there are only a few propagative harmonics in the region between gratings, with the remaining ones being of evanescent nature. Depending on the electrical distance between screens, several evanescent harmonics could have an attenuation constant low enough to give rise to a direct interaction between the two slits discontinuities. In such a case, they should be treated similarly to propagating harmonics, although the characteristic admittance of their equivalent transmission lines would be purely imaginary rather than real. Clearly, as the electrical distance between gratings decreases, more evanescent harmonics would be required to be represented as transmission lines (i.e., as distributed circuit components) connecting the two gratings. This idea was studied in depth by the present authors in [57] in the context of aligned and identical arrays.

Now our attention will focus on the so-called internal region in Fig. 3(b) (gray lines) in order to find an equivalent $\Pi$-circuit topology to describe the field behavior in this region. First, it will be defined as an $n$th internal block, formed by the $n$th left transformer, the $n$th internal transmission line, and the $n$th right transformer. This block admits the following matrix representation in terms of the product of their associated $A B C D$ matrices [60]:

$$
\begin{aligned}
{[A B C D]_{n}=} & {\left[\begin{array}{cc}
\frac{1}{N_{n}\left(w_{1}\right)} & 0 \\
0 & N_{n}\left(w_{1}\right)
\end{array}\right] } \\
& \times\left[\begin{array}{cc}
\cos \left(\beta_{n}^{(1)} d\right) & j Z_{n}^{(1)} \sin \left(\beta_{n}^{(1)} d\right) \\
j Y_{n}^{(1)} \sin \left(\beta_{n}^{(1)} d\right) & \cos \left(\beta_{n}^{(1)} d\right)
\end{array}\right] \\
& \times\left[\begin{array}{cc}
N_{n}\left(w_{2}\right) & 0 \\
0 & \frac{1}{N_{n}\left(w_{2}\right)}
\end{array}\right],
\end{aligned}
$$

where the leftmost (rightmost) matrix represents the $n$th transformer placed at the left-hand (right-hand) termination of the line and the middle matrix refers to the $A B C D$ matrix for the $n$th internal transmission line. After operating, the elements of the resulting $n$th $A B C D$ matrix $\left([A B C D]_{n}\right)$ are found to be

$$
\begin{gathered}
A=\frac{N_{n}\left(w_{2}\right)}{N_{n}\left(w_{1}\right)} \cos \left(\beta_{n}^{(1)} d\right), \\
B=j Z_{n}^{(1)} \frac{1}{N_{n}\left(w_{2}\right) N_{n}\left(w_{1}\right)} \sin \left(\beta_{n}^{(1)} d\right), \\
C=j Y_{n}^{(1)} N_{n}\left(w_{1}\right) N_{n}\left(w_{2}\right) \sin \left(\beta_{n}^{(1)} d\right), \\
D=\frac{N_{n}\left(w_{1}\right)}{N_{n}\left(w_{2}\right)} \cos \left(\beta_{n}^{(1)} d\right) .
\end{gathered}
$$

The $n$th $A B C D$ matrix is next converted into the $n$th admittance matrix $[Y]_{n}$ of a two-port network, whose entries

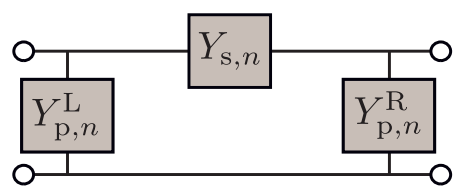

(a)

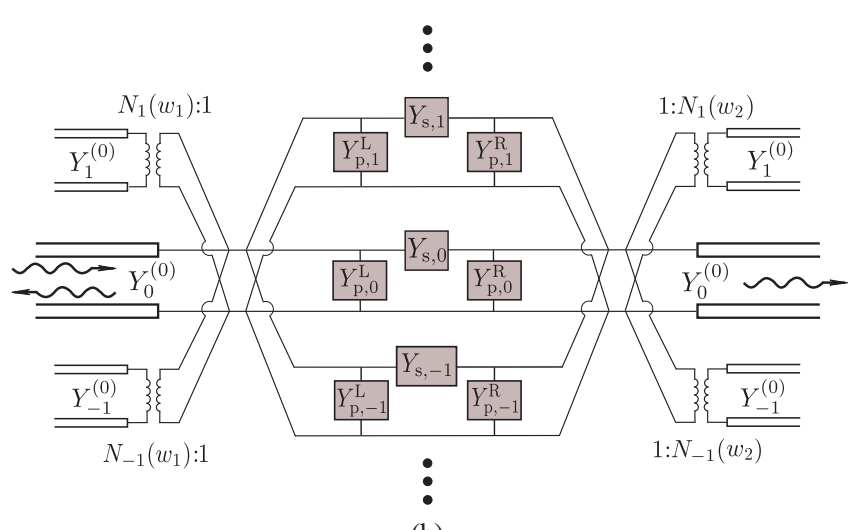

(b)

FIG. 4. (a) The $n$th $\Pi$ block. (b) Equivalent circuit resulting from the substitution of the $\Pi$ blocks. In principle, the circuit consists of an infinite number of $\Pi$ circuits connected in parallel.

are given by

$$
\begin{gathered}
Y_{11}=-j Y_{n}^{(1)} N_{n}^{2}\left(w_{1}\right) \cot \left(\beta_{n}^{(1)} d\right), \\
Y_{12}=j Y_{n}^{(1)} N_{n}\left(w_{1}\right) N_{n}\left(w_{2}\right) \csc \left(\beta_{n}^{(1)} d\right), \\
Y_{21}=j Y_{n}^{(1)} N_{n}\left(w_{2}\right) N_{n}\left(w_{1}\right) \csc \left(\beta_{n}^{(1)} d\right), \\
Y_{22}=-j Y_{n}^{(1)} N_{n}^{2}\left(w_{2}\right) \cot \left(\beta_{n}^{(1)} d\right) .
\end{gathered}
$$

Note that for propagative (evanescent) harmonics, both $Y_{n}^{(1)}$ and $\beta_{n}^{(1)}$ are real (imaginary) and therefore the product $Y_{n}^{(1)} \csc \left(\beta_{n}^{(1)} d\right)$ is always real. In consequence, the present network is reciprocal and lossless $\left(Y_{12}=Y_{21}\right.$ and all the elements of the admittance matrix are imaginary). Thus, the above admittance matrix leads to a conventional $\Pi$ network [60] [see Fig. 4(a)] whose associated series and parallel reactive admittances are given by

$$
\begin{gathered}
Y_{s, n}=-Y_{12}=-j Y_{n}^{(1)} N_{n}\left(w_{1}\right) N_{n}\left(w_{2}\right) \csc \left(\beta_{n}^{(1)} d\right), \\
Y_{p, n}^{L}=Y_{11}+Y_{12}=-j Y_{n}^{(1)} N_{n}^{2}\left(w_{1}\right) \cot \left(\beta_{n}^{(1)} d\right)-Y_{s, n}, \\
Y_{p, n}^{R}=Y_{22}+Y_{12}=-j Y_{n}^{(1)} N_{n}^{2}\left(w_{2}\right) \cot \left(\beta_{n}^{(1)} d\right)-Y_{s, n} .
\end{gathered}
$$

This $n$th $\Pi$ circuit can now substitute the $n$th block previously defined, thus leading to the equivalent network represented in Fig. 4(b). Notice that the internal region is then described by an infinite number of $\Pi$ blocks connected in parallel, where each block represents the contribution of a 
different harmonic in such a region. The parallel admittances of a particular $\Pi$ block $\left(Y_{p, n}^{L}\right.$ and $\left.Y_{p, n}^{R}\right)$ account for the near field around the slit regions, whereas the series admittance $\left(Y_{s, n}\right)$ accounts for the coupling field between both slit arrays associated with the $n$th harmonic (which can be of propagative or evanescent nature). At this point, the same rationale employed in [57] to interpret the physical meaning of the admittances can now be applied. In this sense, the $\Pi$ blocks associated with low-order harmonics will keep the exact dynamical frequency dependence of the modal admittances. The number of low-order harmonics $N$ necessary for the accurate representation of the electrical response is taken according to the criterion discussed in Eq. (16) in [57] (namely, the number of propagative harmonics in the highest-permittivity medium plus the first evanescent one). Since high-order harmonics have a quasistatic frequency dependence, they can be split into two groups depending on the behavior of the series admittance in their corresponding $\Pi$ blocks. The series admittance of high-order blocks accounts for a purely reactive coupling between both screens. For largely spaced gratings, the contribution of this series admittance is practically negligible for all these high-order blocks. On the contrary, for closely spaced arrays, the contribution of the series admittance associated with the lowest-order harmonics of high-order nature can be significant and their contribution has to be explicitly taken into account. The number of high-order harmonics with that significant value of $Y_{s, n}$ is well established by the parameter $M$ previously reported in Eq. (28) in [57].

In summary, the so-called low-order (LO) П blocks keep the dynamical frequency dependence of the modal admittances and they all form a global $\Pi$ block whose admittances are given by

$$
\begin{aligned}
Y_{p, \mathrm{LO}}^{L / R} & =\sum_{n=-N}^{N} Y_{p, n}^{L / R}, \\
Y_{s, \mathrm{LO}} & =\sum_{n=-N}^{N} Y_{s, n} .
\end{aligned}
$$

Similarly, high-order (HO) harmonics also form another global $\Pi$ block. The parallel admittances of this global $\Pi$ block include the contribution of the parallel admittances associated with all the individual high-order $n$th $\Pi$ blocks, but the series admittance of this global high-order $\Pi$ circuit can be formed as the contribution of only a few individual series admittances. It results in the following expressions:

$$
\begin{aligned}
Y_{p, \mathrm{HO}}^{L / R} & =\sum_{\forall|n| \geqslant N+1}^{\infty} Y_{p, n}^{L / R}, \\
Y_{s, \mathrm{HO}} & =\sum_{\forall|n| \geqslant N+1}^{|M|} Y_{s, n} .
\end{aligned}
$$

Certainly, for TM incidence, the admittances in (20) and (21) become capacitive and, for TE incidence, they become inductive. The final version of the equivalent circuit for both TM and TE incidence is illustrated in Fig. 5.

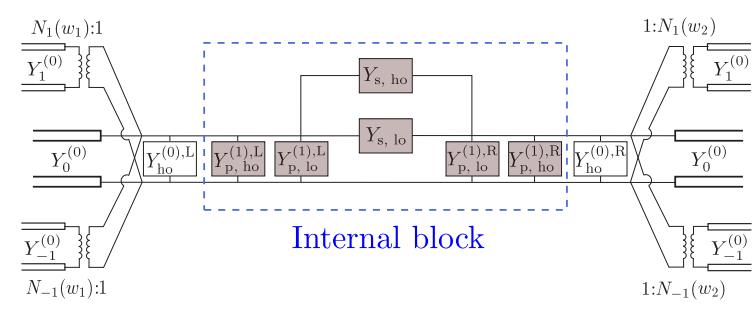

FIG. 5. Final version of the equivalent circuit.

In this figure we have boxed the block containing all the information about the internal region (internal block). The external region is characterized by the high-order admittance $Y_{\mathrm{HO}}^{(0)}$, which accounts for the reactive field around the external face of the slits gratings, in addition to some transmission lines accounting for the low-order fields. Following a procedure similar to that in [57], internal blocks can now be cascaded to build up an equivalent circuit for dealing with a stack of an arbitrary number of slit arrays.

\section{B. Equivalent $\Pi$ topology for two different and nonaligned coupled gratings}

In the previous section, an equivalent circuit was derived for a pair of strongly coupled nonidentical but aligned arrays (the aligned gratings have different slit widths but the same period and the same relative location of the slits in the unit cell). Nonaligned arrays are also of interest in the literature, as reported in $[55,59,61]$. The general methodology described above can still be employed to deal with the nonaligned case, although the authors could derive a compact $\Pi$ circuit for the internal region only for the case of normal incidence.

A sketch of the system under consideration is shown in Fig. 6(a) together with its corresponding unit cell in Fig. 6(b). The misalignment between the centers of both slit apertures (within the same unit cell) is given by $h$. The same procedure in [38] can also be applied to vertically displaced single slit gratings, resulting in the same circuit model as for centered slit gratings (Fig. 2 above), but with the difference that now the transformer turn ratios are complex numbers given by

$$
\bar{N}_{n}\left(w_{i}, h_{i}\right)=N_{n}\left(w_{i}\right) e^{j k_{n} h_{i}}
$$

where $N_{n}\left(w_{i}\right)$ are the real-valued ratios in (4). The additional phase factor $e^{j k_{n} h_{i}}$ appearing in (22) comes from the vertical displacement of the slits, since now the Fourier integral of the aperture field is evaluated in an interval that is not symmetric with respect to $y=0$. This can be interpreted as an ideal transformer, which, in addition to the usual scale factor, also introduces a phase shift in the output voltage and current with respect to the corresponding input magnitudes. Such an ideal complex transformer is lossless and should not store any reactive energy either. Taking into account these considerations, the $A B C D$ matrix for the ideal complex transformer is

$$
[A B C D]_{1: \bar{N}}=\left[\begin{array}{cc}
1 / \bar{N} & 0 \\
0 & \bar{N}^{*}
\end{array}\right] .
$$



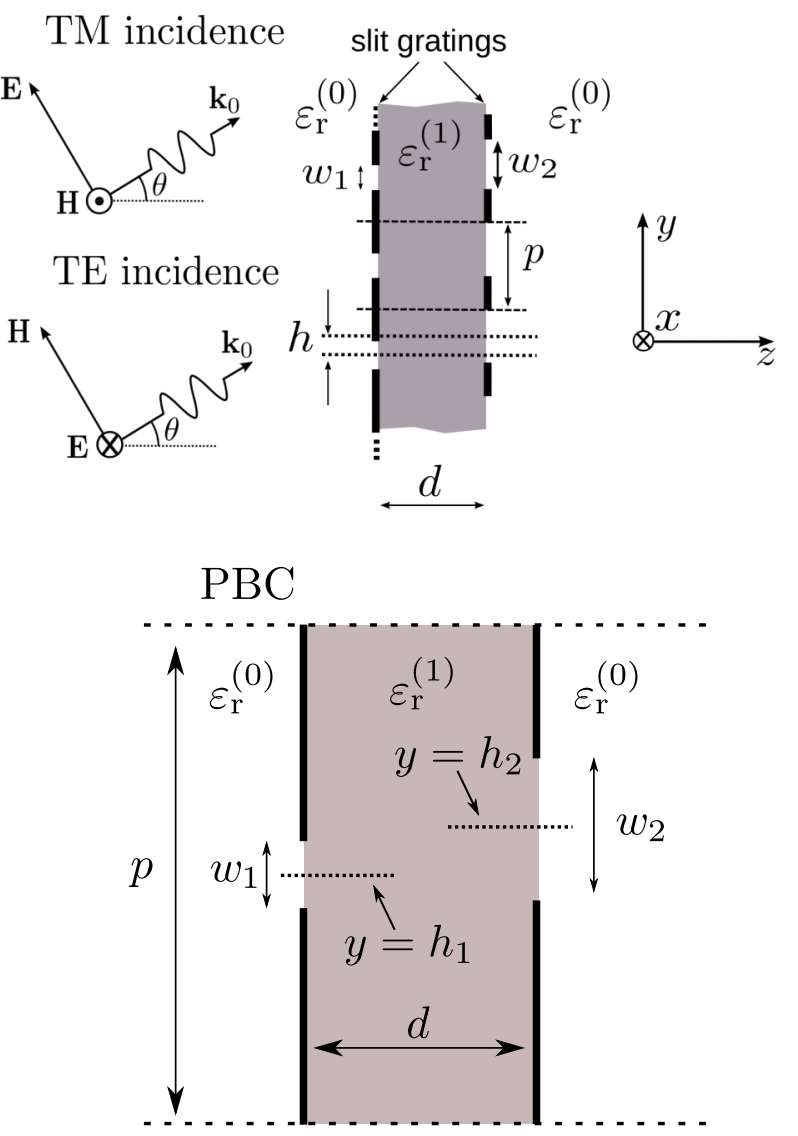

PBC

FIG. 6. (a) Two coupled nonaligned slit gratings. (b) Unit cell of the problem. The centers of the slits are placed at heights $h_{1}$ and $h_{2}$ with respect to an arbitrary $y$ axis zero, with $h=h_{2}-h_{1}$.

Proceeding as in the previous section, the multimode equivalent circuit for the complete coupled structure is readily obtained by connecting the corresponding $n$th lines in the single-grating circuits, as shown in Fig. 7. The elements of the admittance matrix $[Y]_{n}$ corresponding to the internal system

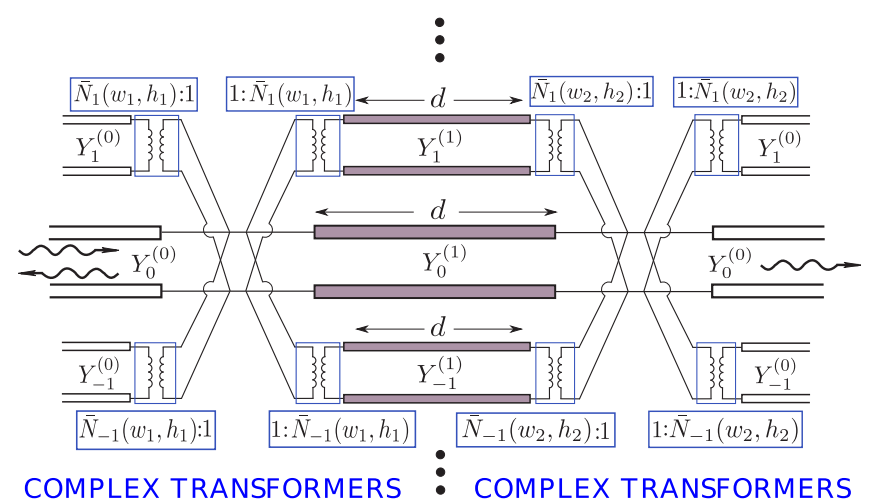

FIG. 7. Equivalent circuit corresponding to a pair of coupled and nonaligned slits arrays. The turn ratios of the transformers are given by the complex-valued expression in (22). formed by the $n$th left or right transformer and the $n$th internal line are now found to be given by

$$
\begin{gathered}
Y_{11, n}=-j Y_{n}^{(1)} N_{n}^{2}\left(w_{1}\right) \cot \left(\beta_{n}^{(1)} d\right), \\
Y_{12, n}=j Y_{n}^{(1)} N_{n}\left(w_{1}\right) N_{n}\left(w_{2}\right) e^{j k_{n} h} \csc \left(\beta_{n}^{(1)} d\right), \\
Y_{21, n}=j Y_{n}^{(1)} N_{n}\left(w_{2}\right) N_{n}\left(w_{1}\right) e^{-j k_{n} h} \csc \left(\beta_{n}^{(1)} d\right), \\
Y_{22, n}=-j Y_{n}^{(1)} N_{n}^{2}\left(w_{2}\right) \cot \left(\beta_{n}^{(1)} d\right),
\end{gathered}
$$

with $h=h_{2}-h_{1}$. Since the product $Y_{n}^{(1)} \csc \left(\beta_{n}^{(1)} d\right)$ is always real, these admittance matrices are skew Hermitian $\left(Y_{i j}=-Y_{j i}^{*}\right)$, which is the general condition for lossless networks [62]. However, since the off-diagonal elements are not purely imaginary, now the matrices are not symmetric, which means that the network cannot be represented in terms of regular, bilateral admittances (in either a $\Pi$ network or any other network topology). Nevertheless, it is still feasible to build the global admittance matrix of the internal region from the summation of all the individual $n$th admittance matrices:

$$
[Y]=\sum_{n=-\infty}^{\infty}\left[Y_{n}\right],
$$

where the entries of the $\left[Y_{n}\right]$ matrix are given by (24)-(27). This admittance matrix can be straightforwardly converted into its associated scattering matrix [60]. In particular, the off-diagonal terms (transmission coefficients) can be written as

$$
\begin{aligned}
& S_{12}=\frac{-2 Y_{0}^{(0)}}{\Delta Y} Y_{12}, \\
& S_{21}=\frac{-2 Y_{0}^{(0)}}{\Delta Y} Y_{21},
\end{aligned}
$$

with

$$
\Delta Y=\left(Y_{11}+Y_{0}^{(0)}\right)\left(Y_{22}+Y_{0}^{(0)}\right)-Y_{12} Y_{21}
$$

Note that, since $Y_{12} \neq Y_{21}$, the transmission coefficients $S_{12}$ and $S_{21}$ provided by the equivalent network are not equal (although $Y_{12}=-Y_{21}^{*}$ does imply $\left|S_{12}\right|=\left|S_{21}\right|$, as expected since the obtained circuit is lossless [62]). In this sense, the obtained equivalent network is not reciprocal. However, it should be emphasized that this is by no means a consequence of an actual nonreciprocal behavior of the considered coupled-grating structure, which is of course reciprocal. The explanation for this apparent inconsistency is given in the Appendix.

The above discussion does not apply to the case of normal incidence. In this case $k_{t}=0$ and then harmonics of opposite orders in the internal region satisfy

$$
\begin{aligned}
& \beta_{n}^{(1)}=\beta_{-n}^{(1)}, \\
& Y_{n}^{(1)}=Y_{-n}^{(1)}, \\
& N_{n}=N_{-n} .
\end{aligned}
$$


Taking advantage of (32)-(34), we can construct the following $n$th modal admittance matrix by grouping harmonics with opposite orders $\left(\hat{Y}_{i j, n}=Y_{i j, n}+Y_{i j,-n}\right)$ :

$$
\begin{gathered}
\hat{Y}_{11, n}=-2 j Y_{n}^{(1)} N_{n}^{2}\left(w_{1}\right) \cot \left(\beta_{n}^{(1)} d\right), \\
\hat{Y}_{12, n}=2 j Y_{n}^{(1)} N_{n}\left(w_{1}\right) N_{n}\left(w_{2}\right) \cos \left(k_{n} h\right) \csc \left(\beta_{n}^{(1)} d\right), \\
\hat{Y}_{21, n}=2 j Y_{n}^{(1)} N_{n}\left(w_{2}\right) N_{n}\left(w_{1}\right) \cos \left(k_{n} h\right) \csc \left(\beta_{n}^{(1)} d\right), \\
\hat{Y}_{22, n}=-2 j Y_{n}^{(1)} N_{n}^{2}\left(w_{2}\right) \cot \left(\beta_{n}^{(1)} d\right) .
\end{gathered}
$$

Since this resulting modal admittance matrix is symmetric, a conventional $\Pi$ network can be derived whose series and parallel admittances are given by

$$
\begin{gathered}
Y_{s, n}=-2 j Y_{n}^{(1)} N_{n}\left(w_{1}\right) N_{n}\left(w_{2}\right) \cos \left(k_{n} h\right) \csc \left(\beta_{n}^{(1)} d\right), \\
Y_{p, n}^{L}=-2 j Y_{n}^{(1)} N_{n}^{2}\left(w_{1}\right) \cot \left(\beta_{n}^{(1)} d\right)-Y_{s, n}, \\
Y_{p, n}^{R}=-2 j Y_{n}^{(1)} N_{n}^{2}\left(w_{2}\right) \cot \left(\beta_{n}^{(1)} d\right)-Y_{s, n} .
\end{gathered}
$$

The infinite number of $\Pi$ blocks admits a decomposition into low-order and high-order blocks, as it was discussed for the aligned-grating case. The admittances in the low-order blocks retain their dynamical frequency dependence. The parallel connection of the low-order $\Pi$ blocks gives rise to a global $\Pi$ block whose elements are defined as

$$
\begin{aligned}
Y_{p, \mathrm{LO}}^{L / R} & =\sum_{n=0}^{N} Y_{p, n}^{L / R}, \\
Y_{s, \mathrm{LO}} & =\sum_{n=0}^{N} Y_{s, n} .
\end{aligned}
$$

The $\Pi$ blocks associated with high-order harmonics also give rise to a global $\Pi$ circuit whose parallel admittances are the result of the contribution of all the individual parallel admittances of the high-order $\Pi$ blocks, whereas the series admittance of the global $\Pi$ circuit is the result of the contribution of a few series admittances,

$$
\begin{aligned}
Y_{p, \mathrm{HO}}^{L / R} & =\sum_{n=N+1}^{\infty} Y_{p, n}^{L / R}, \\
Y_{s, \mathrm{HO}} & =\sum_{n=N+1}^{M} Y_{s, n} .
\end{aligned}
$$

The admittances in (44) and (45) are purely capacitive for TM incidence and purely inductive for TE incidence. The resulting equivalent circuit has the same topology as the one depicted in Fig. 5.

\section{NUMERICAL RESULTS}

In order to check the robustness and accuracy of the proposed approach, some results are shown in this section. The first example can be found in Fig. 8, where three different plots represent the reflection coefficient obtained for three different structures under normal TM incidence. Figure 8(a) shows the reflection response of a pair of two aligned and

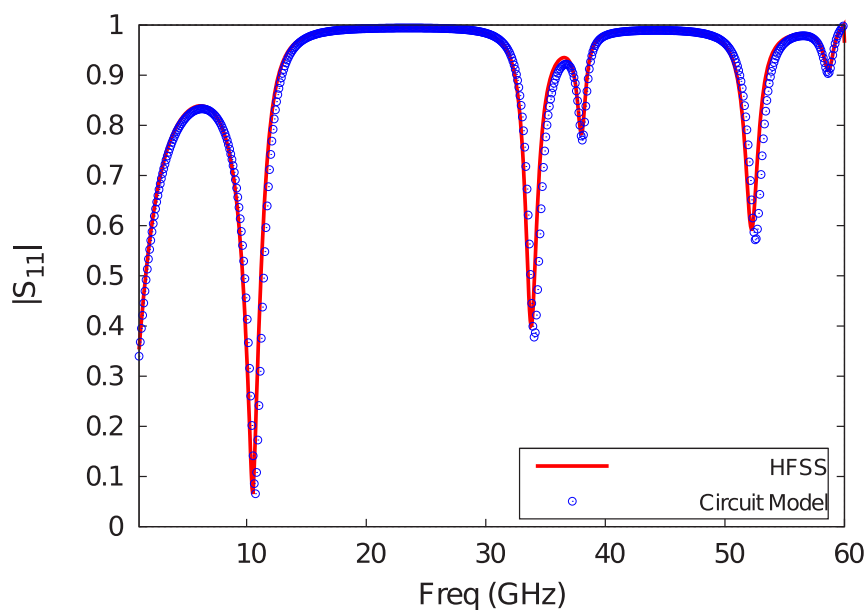

(a)

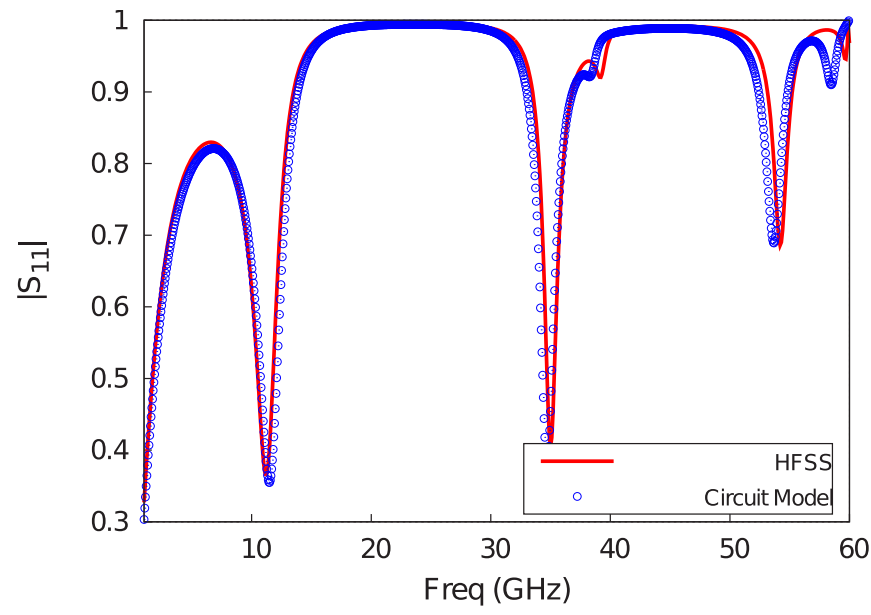

(b)

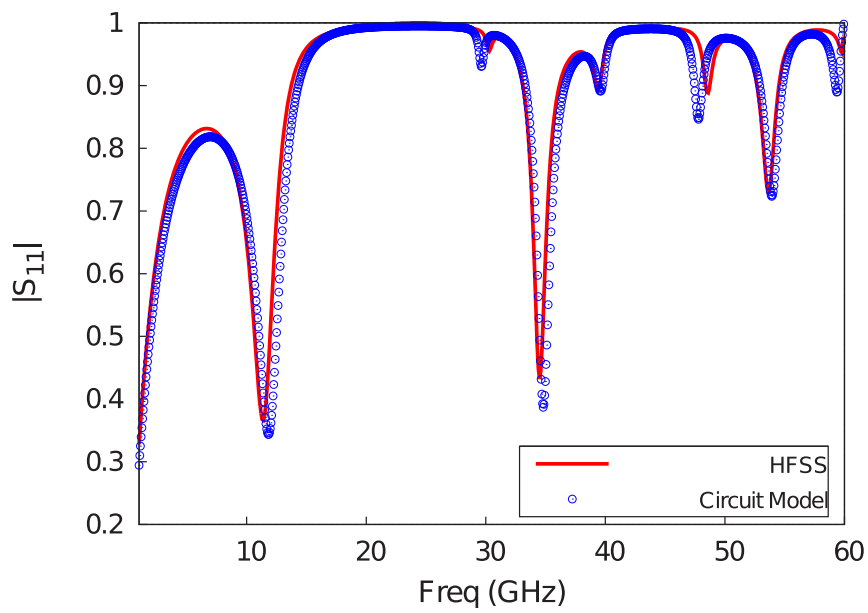

(c)

FIG. 8. Reflection coefficient versus frequency under TM normal incidence for three cases. (a) A coupled and aligned pair of identical slit arrays. The structure parameters are $p=5 \mathrm{~mm}$, $w_{1}=w_{2}=0.5 \mathrm{~mm}, \varepsilon_{r}^{(0)}=1, \varepsilon_{r}^{(1)}=4.4-j 0.088, d=2 \mathrm{~mm}$, and $h=0 \mathrm{~mm}$. (b) A coupled and aligned pair of different slit arrays. The structure parameters are the same as in (a) except for $w_{2}=1 \mathrm{~mm}$. (c) A coupled and nonaligned pair of different slit arrays. The structure parameters are the same as in (b) except for $h=1 \mathrm{~mm}$. 


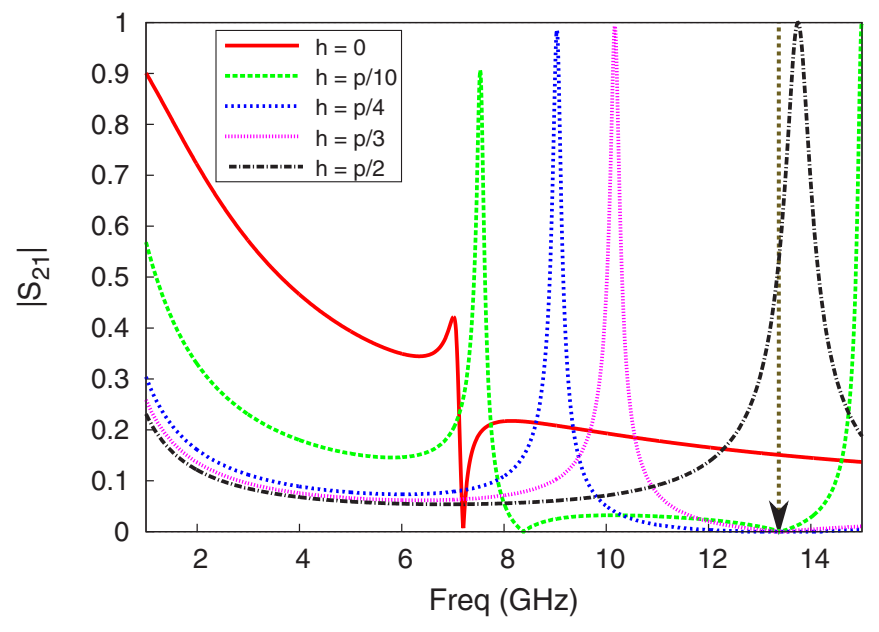

FIG. 9. Transmission coefficient versus frequency obtained from the configuration in Fig. 6(b) under normal TM incidence. Five different values of $h$ have been regarded. The vertical dotted line in brown indicates the frequency of Wood's anomaly in the internal medium, at $13.42 \mathrm{GHz}$. The structure parameters are $p=10 \mathrm{~mm}$, $w_{1}=0.3 \mathrm{~mm}, w_{2}=1 \mathrm{~mm}, d=0.3 \mathrm{~mm}, \varepsilon_{r}^{(0)}=1$, and $\varepsilon_{r}^{(1)}=5$.

identical coupled arrays sandwiching a FR4-dielectric layer of thickness $d=2 \mathrm{~mm}$. Assuming this same structure, but modifying the slit size of the rightmost array $\left(w_{2}\right)$, its reflection coefficient is plotted in Fig. 8(b). The third structure analyzed in Fig. 8(c) is the same as the one in Fig. 8(b), but shifting the rightmost array $1 \mathrm{~mm}$ upward. The influence of the geometrical modifications in the reflection spectra can be observed, especially at high frequencies. There is a reflection minimum close to $10 \mathrm{GHz}$ that appears for all the structures. The aligned cases in Figs. 8(a) and 8(b) show three reflections dips beyond $30 \mathrm{GHz}$ located, approximately, at the same frequencies. On the contrary, the nonaligned case provides an additional reflection minimum. The agreement with results from HFSS [58] is quite good in all cases. It should be noticed that, due to the analytical nature of the proposed circuit model, the CPU time employed to generate our data in the above figures is almost negligible in comparison with the one required by HFSS.

As mentioned above, misaligned arrays can be employed for tailoring the transmission properties of an incident plane wave when the arrays are closely spaced. For the structure depicted in Fig. 6, five curves are plotted in Fig. 9, each one representing the transmission coefficient obtained for a different value of $h$. A transmission maximum is observed in all the curves. This transmission maximum moves to higher frequencies as $h$ increases. Note that for $h<p / 4$ the transmission peak does not reach unity (perfect transmission) and it is followed by a transmission dip (full reflection). For $p / 4 \leqslant h \leqslant p / 2$, full transmission is achieved but full reflection only appears at the frequency associated with Wood's anomaly in the dielectric medium at $13.42 \mathrm{GHz}$. The reasons behind the appearance of full reflection just after the transmission peak can be explained in terms of the equivalent circuit. A simplified version of the model is represented in Fig. 10 and, more specifically, the equivalent circuit obtained for coupled gratings misaligned with $h<p / 4$ is shown in

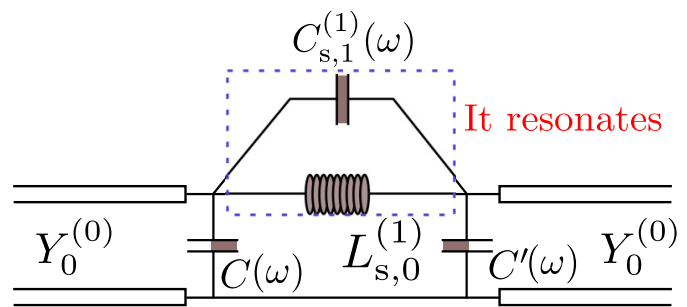

(a)

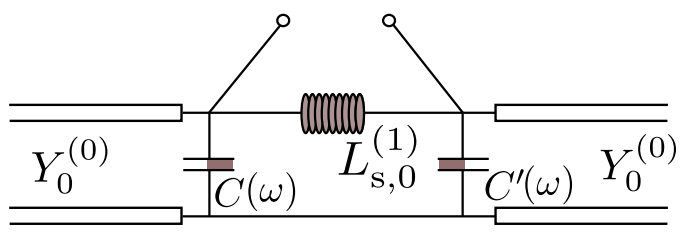

(b)

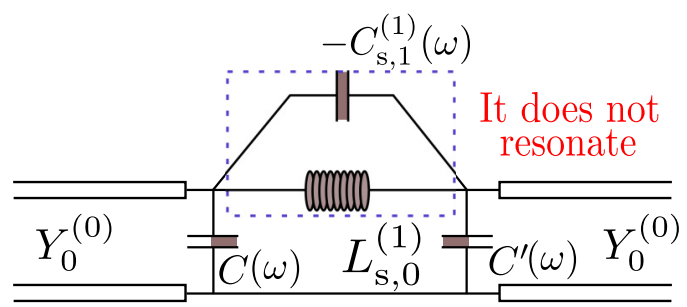

(c)

FIG. 10. Topology of the $\Pi$ circuit as a function of $h$. (a) Topology of the $\Pi$ network for a misalignment of $h<p / 4$, where $C_{s, 1}^{(1)}(\omega)$ remains positive. (b) Equivalent circuit for $h=p / 4$. Now the capacitance associated with the first-order mode is null. (c) Equivalent circuit for $p / 4 \leqslant h \leqslant p / 2$, where the capacitance $C_{s, 1}^{(1)}(\omega)$ has become negative.

Fig. 10(a). The relevant series elements of interest are assumed to be those associated with the fundamental and first-order modes. According to (39), the series element associated with the zeroth-order mode can be recognized as the following inductive admittance:

$$
Y_{s, 0}=-2 j Y_{0}^{(1)} \csc \left(\beta_{0}^{(1)} d\right) \approx-j \frac{2}{\mu_{0} \omega d},
$$

where $\sin \left(\beta_{0}^{(1)} d\right)$ has been approximated as $\beta_{0}^{(1)} d$, leading to an inductance of value $L_{s, 0}^{(1)}=\mu_{0} d / 2$. Similarly, the series element associated with the first-order mode is given by

$$
\begin{aligned}
Y_{s, 1} & =-2 j Y_{1}^{(1)} N_{1}\left(w_{1}\right) N_{1}\left(w_{2}\right) \cos \left(k_{1} h\right) \csc \left(\beta_{1}^{(1)} d\right) \\
& \approx j \omega \frac{2 \varepsilon_{0} \varepsilon_{r} N_{1}\left(w_{1}\right) N_{1}\left(w_{2}\right) \cos \left(k_{1} h\right)}{\left[\beta_{1}^{(1)}\right]^{2} d}=j \omega C_{s, 1}^{(1)}(\omega) .
\end{aligned}
$$

When the $L C$ tank formed by $C_{s, 1}^{(1)}(\omega)$ and $L_{s, 0}^{(1)}$ resonates, it gives rise to an open circuit that causes full reflection in the circuit. For frequencies below such a resonance, a 


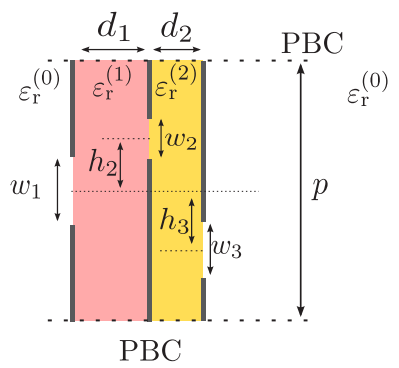

(a)

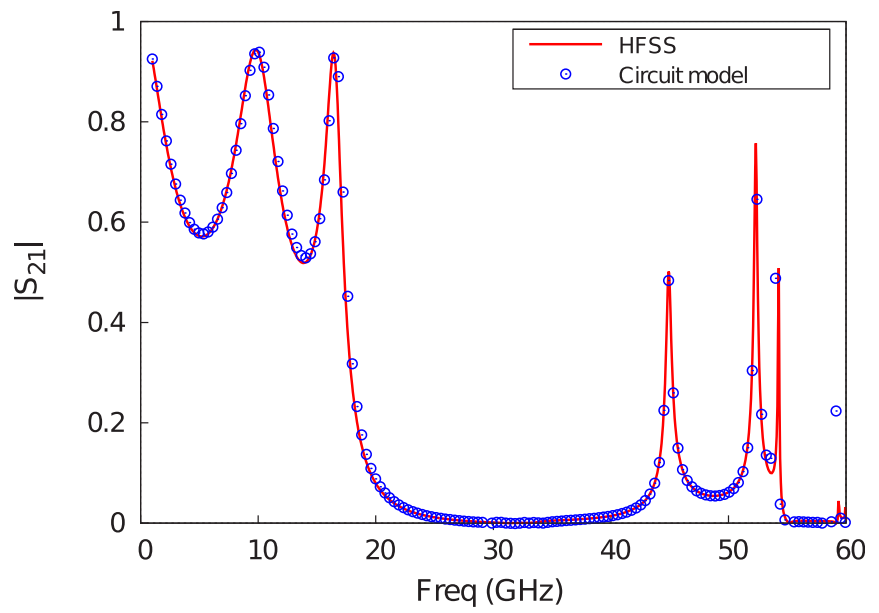

(b)

FIG. 11. (a) Unit cell of the structure under analysis. (b) Transmission coefficient versus frequency for the structure in (a) for TM normal incidence. The structure parameters are $p=5 \mathrm{~mm}, w_{1}=$ $0.5 \mathrm{~mm}, w_{2}=1 \mathrm{~mm}, w_{3}=0.8 \mathrm{~mm}, d_{1}=2 \mathrm{~mm}, d_{2}=1.5 \mathrm{~mm}$, $h_{2}=0 \mathrm{~mm}, h_{3}=1.5 \mathrm{~mm}, \varepsilon_{r}^{(0)}=1, \varepsilon_{r}^{(1)}=2.2$, and $\varepsilon_{r}^{(2)}=4$.

transmission peak appears as a result of the global resonance given among the parallel admittances $C(\omega)$ and $C^{\prime}(\omega)$ and the series admittances, with

$$
\begin{aligned}
& j \omega C(\omega)=Y_{\mathrm{HO}}^{(0)}+Y_{p, \mathrm{HO}}^{L}+Y_{p, \mathrm{LO}}^{L}, \\
& j \omega C^{\prime}(\omega)=Y_{\mathrm{HO}}^{(0)}+Y_{p, \mathrm{HO}}^{R}+Y_{p, \mathrm{LO}}^{R} .
\end{aligned}
$$

It is important to highlight that $C_{s, 1}^{(1)}(\omega)$ behaves as a positive capacitance for $h<p / 4$. When $h=p / 4$, it can be inferred from (47) and observed in Fig. 10(b) that $C_{s, 1}^{(1)}(\omega)=0$. Hence, no series resonance is expected and no full reflection appears. The shift of the transmission peak toward higher frequencies as $h$ increases can be explained in terms of the decrease of $C_{s, 1}^{(1)}(\omega)$ from $h=0$ to $h=p / 4$. This decrease in the series capacitance reduces the global capacitance in the $\Pi$ network and increases the global resonance frequency associated with the transmission peak. For $p / 4 \leqslant h \leqslant p / 2$, the capacitance $C_{s, 1}^{(1)}(\omega)$ becomes negative, enhancing the "inductive" contribution of the series elements and therefore increasing the frequency of the transmission resonance of the circuit. The series branch formed by $L_{s, 0}^{(1)}$ and $-C_{s, 1}^{(1)}(\omega)$ does not resonate [Fig. 10(c)].

Another example is shown in Fig. 11 and consists of a stack of three different and nonaligned slit arrays [Fig. 11(a)] excited by a normally impinging TM plane wave. This configuration

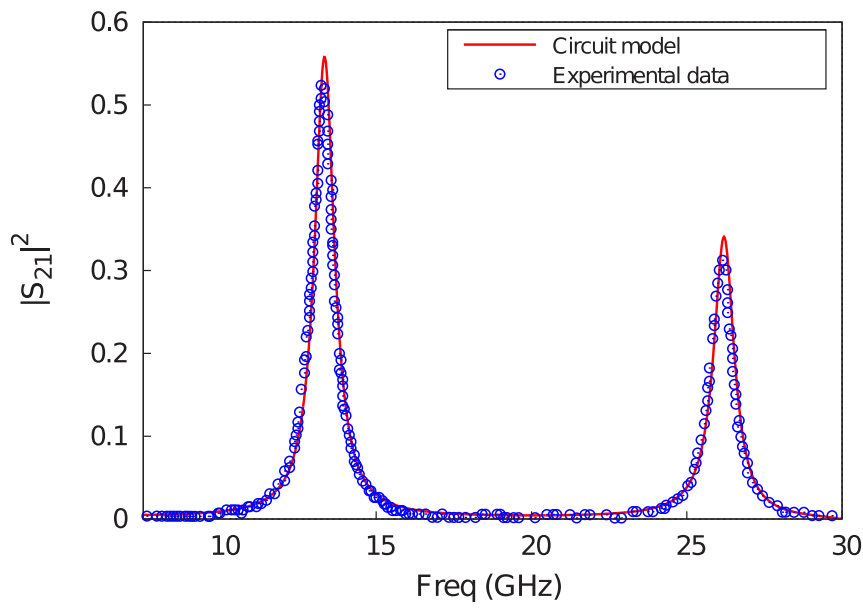

FIG. 12. Transmissivity versus frequency. Comparison of the results from the model with experimental data extracted from [59]. Results were obtained for a pair of two coupled and nonaligned slit arrays. The structure parameters are $p=10.01 \mathrm{~mm}$, $w_{1}=w_{2}=0.33 \mathrm{~mm}, \quad h=p / 2, \quad d=0.356 \mathrm{~mm}, \quad \varepsilon_{r}^{(0)}=1, \quad$ and $\varepsilon_{r}^{(1)}=4.17-j 0.07$.

provides a quite complicated transmission spectrum, as shown in Fig. 11(b). It is remarkable that the analytical equivalent circuit matches very well the numerical results provided by HFSS. It is worth mentioning that, at the highest frequency of operation $(60 \mathrm{GHz})$, there are two propagative modes inside the denser dielectric $\left(\varepsilon_{r}^{(2)}=4\right)$.

Figure 12 shows a comparison of the results provided by our model vs experimental data reported in [59]. The plotted transmissivity coefficient corresponds to a pair of identical nonaligned slit arrays under normal TM incidence (the gratings are printed on both faces of a lossy FR4-dielectric slab). Even though two transmission maxima appear around 13 and $26 \mathrm{GHz}$, no full transmission is achieved due to the losses associated with the dielectric layer. The inclusion of losses in the dielectric is straightforwardly incorporated in the model by using the appropriate complex-valued permittivity of the dielectric. The agreement shown between both curves is excellent.

Finally, a last example is shown in Fig. 13 for a pair of nonidentical and nonaligned coupled gratings under oblique TE or TM incidence (structure in Fig. 6). In this situation, as explained previously, there is not an equivalent $\Pi$ circuit for the structure. Thus, the scattering parameters of the structure are computed after calculating the network admittance matrix. The figure shows a comparison of the magnitude of the transmission coefficient provided by our circuit model and by HFSS. Very good agreement between both sets of data can be observed in Fig. 13(a) in the frequency range under consideration. This particular plot deals with TE incidence, where the structure is practically opaque except for some narrow frequency intervals, associated with the excitation of higher-order modes and Fabry-Pérot resonances in the dielectric medium, $\varepsilon_{r}^{(1)}=9$. The case of TM incidence plotted in Fig. 13(b) also shows good agreement with results from HFSS, although some differences can be observed around $50 \mathrm{GHz}$. 


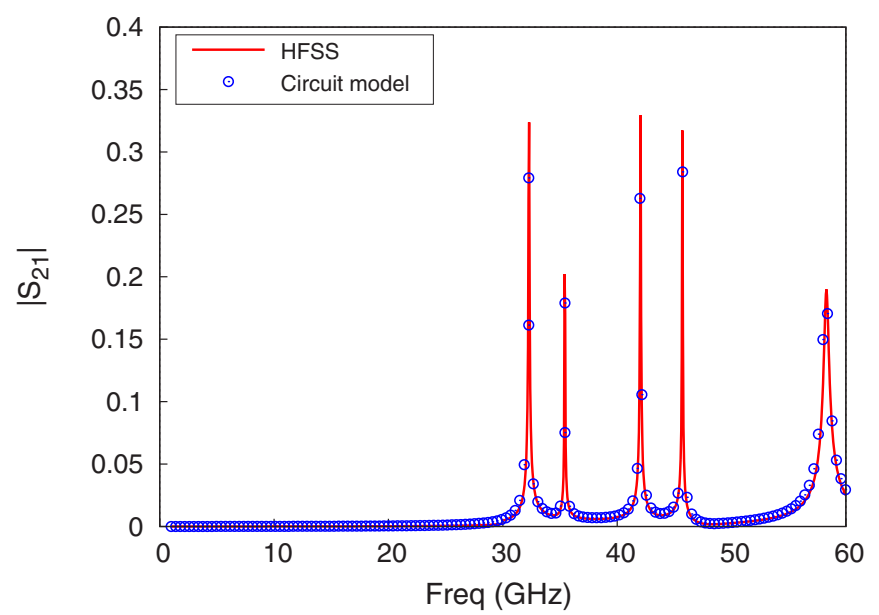

(a)

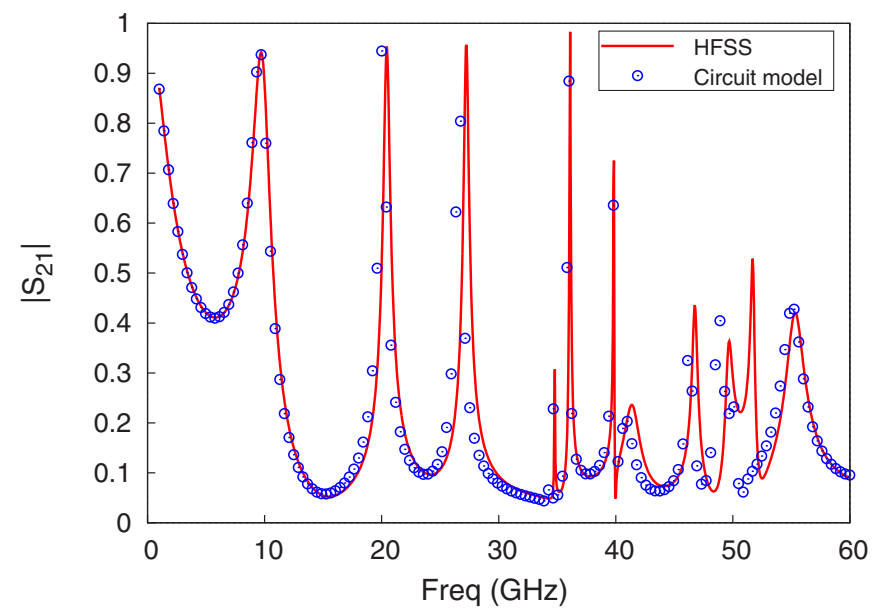

(b)

FIG. 13. Transmission coefficient versus frequency for an obliquely incident wave: (a) TE incidence and (b) TM incidence. The structure parameters are $p=5 \mathrm{~mm}, w_{1}=1 \mathrm{~mm}, w_{2}=0.5 \mathrm{~mm}$, $d=1.5 \mathrm{~mm}, h=1 \mathrm{~mm}, \varepsilon_{r}^{(0)}=1, \varepsilon_{r}^{(1)}=9$, and $\theta=30^{\circ}$.

\section{CONCLUSION}

An equivalent $\Pi$ network has been derived to compute the scattering properties of a pair of nonidentical coupled slit arrays with the same period. The equivalent circuit accounts for normal and oblique TE and TM incidence in the case of nonidentical but aligned slit arrays. The equivalent $\Pi$ network also accounts for the scattering of nonaligned and coupled arrays, but only for normal incidence. No П network has been found for oblique incidence assuming nonaligned arrays due to the fact that the obtained admittance matrices are not symmetric. This lack of symmetry comes from the natural appearance of phase-shifting transformers with complex turn ratios. The scattering parameters in this case can be calculated directly from the network matrix formalism. Fully analytical expressions are obtained for all the circuit elements, making the model self-contained and very efficient from a computational point of view. The model provides physical insight into the behavior of the structure, thus helping to easily understand the appearance and the displacement of transmission and reflection peaks. In addition, it can be straightforwardly extended to obtain the scattering parameters of stacked arrays.

\section{ACKNOWLEDGMENTS}

This work was supported by the Spanish Ministerio de Economía y Competitividad with European Union FEDER funds (Project No. TEC2013-41913-P) and by the Consejería de Economía y Conocimiento, Spanish Junta de Andalucía (Project No. P12-TIC-1435).

\section{APPENDIX: NONRECIPROCITY OF THE CIRCUIT MODEL}

The reciprocity theorem for the electromagnetic field in a source-free region states that

$$
\oint_{S}\left(\mathbf{E}^{(a)} \times \mathbf{H}^{(b)}-\mathbf{E}^{(b)} \times \mathbf{H}^{(a)}\right) \cdot d \mathbf{S}=0,
$$

where $S$ is the surface enclosing the considered region and the superscripts refer to two independent excitations $a$ and $b$. If the considered region is a given waveguide section, then we can split the integration surface into the input and output ports $(P)$ and the lateral walls of the waveguide $(L)$ :

$$
\begin{aligned}
& \int_{P}\left(\mathbf{E}^{(a)} \times \mathbf{H}^{(b)}-\mathbf{E}^{(b)} \times \mathbf{H}^{(a)}\right) \cdot d \mathbf{S} \\
& \quad+\int_{L}\left(\mathbf{E}^{(a)} \times \mathbf{H}^{(b)}-\mathbf{E}^{(b)} \times \mathbf{H}^{(a)}\right) \cdot d \mathbf{S}=0 .
\end{aligned}
$$

For an actual metallic waveguide or a virtual waveguide bounded by perfect electric or magnetic walls (discontinuities may exist within the considered waveguide section), the lateral integrals vanish because the tangential electric field, or magnetic field in the case of a virtual magnetic wall, is zero. In this case, reciprocity guarantees that the integrals of the $a$ and $b$ fields at the two ports $P_{1}$ and $P_{2}$ satisfy

$$
\begin{aligned}
& \int_{P_{1}}\left(\mathbf{E}^{(a)} \times \mathbf{H}^{(b)}-\mathbf{E}^{(b)} \times \mathbf{H}^{(a)}\right) \cdot d \mathbf{S} \\
& \quad+\int_{P_{2}}\left(\mathbf{E}^{(a)} \times \mathbf{H}^{(b)}-\mathbf{E}^{(b)} \times \mathbf{H}^{(a)}\right) \cdot d \mathbf{S}=0 .
\end{aligned}
$$

It is easy to show (see, for instance, [60]) that (A3) directly implies that the transmission coefficients between the two ports are the same in either direction $\left(S_{12}=S_{21}\right)$ and thus the considered waveguide problem can be modeled, in principle, using a reciprocal equivalent circuit (i.e., an equivalent circuit with symmetric scattering and admittance matrices).

In the case under study in this work, the virtual waveguide is not bounded by perfect electric or magnetic walls but by periodic boundary conditions that, for a given incidence angle, impose a fixed phase shift between the fields at the boundaries of the unit cell waveguide. Thus, let us consider excitations $a$ and $b$ as plane waves impinging with an incidence angle $\theta$ from either side of the structure, as shown in Fig. 14.

For both excitations, the fields at the lower (say, $y=0$ ) and upper $(y=p)$ unit cell boundaries are related in the following way:

$$
\left.\mathbf{A}^{(a / b)}\right|_{y=p}=\left.e^{-j k_{t} p} \mathbf{A}^{(a / b)}\right|_{y=0},
$$



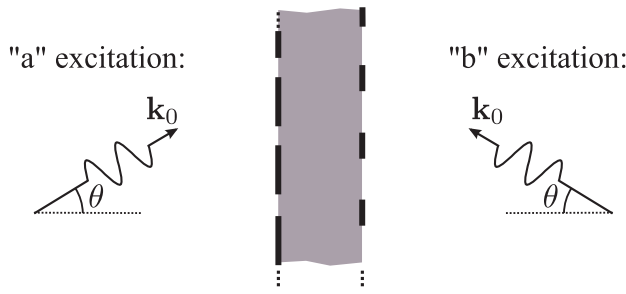

FIG. 14. Two plane-wave excitations of the coupled gratings, impinging from different sides but with the same incidence angle $\theta$. For $\theta \neq 0$, the transmission coefficients for the $a$ and $b$ excitations have the same magnitude but different phase. This is the scenario modeled by our equivalent network.

where $\mathbf{A}$ stands for either $\mathbf{E}$ or $\mathbf{H}$. Each term in the lateral integrals (per unit length in the $x$ direction) can then be written as

$$
\begin{gathered}
\int_{y=0}\left(\mathbf{E}^{(a / b)} \times \mathbf{H}^{(b / a)}\right) \cdot(-\hat{\mathbf{y}}) d z+\int_{y=p}\left(\mathbf{E}^{(a / b)} \times \mathbf{H}^{(b / a)}\right) \cdot \hat{\mathbf{y}} d z \\
=\left(e^{-2 j k_{t} p}-1\right) \int_{y=0}\left(\mathbf{E}^{(a / b)} \times \mathbf{H}^{(b / a)}\right) \cdot \hat{\mathbf{y}} d z .
\end{gathered}
$$

In general, the contribution of the lateral integrals is not zero, which implies that (A3) is not satisfied and, consequently, $S_{21} \neq S_{12}$. Nevertheless, it is not possible that the transmission coefficients of a lossless structure have different magnitude [62]. This fact causes the appearance of the phase-shifting (complex) transformers in our circuit model. For normal incidence, however, $k_{t}=0$ and therefore (A5) is identically zero. In this case Eq. (A3) does hold and it is indeed possible to derive an explicit $\Pi$-network topology for the equivalent circuit.

Finally, it is interesting to consider the case in which the structure is excited by plane waves that impinge with opposite angles, as shown in Fig. 15. This might seem a more natural scenario when considering reciprocity in the complete
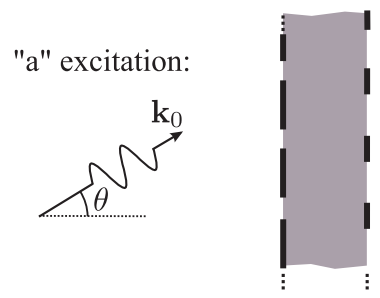

"b" excitation:

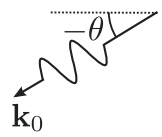

FIG. 15. Two plane-wave excitations impinging from different sides and with opposite incidence angle. The transmission coefficients for the $a$ and $b$ excitations have the same complex value (magnitude and phase).

electromagnetic problem. In this situation we have

$$
\begin{aligned}
& \left.\mathbf{A}^{(a)}\right|_{y=p}=\left.e^{-j k_{t} p} \mathbf{A}^{(a)}\right|_{y=0}, \\
& \left.\mathbf{A}^{(b)}\right|_{y=p}=\left.e^{+j k_{t} p} \mathbf{A}^{(b)}\right|_{y=0},
\end{aligned}
$$

which, because of the different signs in the phase shifts, clearly implies that

$$
\left[\mathbf{E}^{(a / b)} \times \mathbf{H}^{(b / a)}\right]_{y=p}=\left[\mathbf{E}^{(a / b)} \times \mathbf{H}^{(b / a)}\right]_{y=0}
$$

and hence the lateral integrals in the upper and lower unit cell boundaries cancel out and (A3) is satisfied once again. In consequence, the transmission coefficients for the $a$ and $b$ excitations in Fig. 15 have the same complex value (magnitude and phase). The reader can verify that our model is consistent with this observation since it implies $S_{21}(\theta)=S_{12}(-\theta)$. A relevant consequence of this fact is that, for the aligned coupled gratings considered in Sec. II A, the transmission coefficients for the $a$ and $b$ excitations in Fig. 14 also have the same complex value. Indeed, for vertically aligned gratings, the symmetry of the structure with respect to the slits' middle plane (see Fig. 1) clearly implies that the problem is invariant with respect to a change of sign in the incidence angle and therefore $S_{21}(\theta)=S_{12}(-\theta)=S_{12}(\theta)$.
[1] R. W. Wood, Proc. Phys. Soc. London 18, 269 (1902).

[2] L. Rayleigh, Philos. Mag. 14, 60 (1907).

[3] R. W. Wood, Phys. Rev. 48, 928 (1935).

[4] T. W. Ebbesen, H. J. Lezec, H. F. Ghaemi, T. Thio, and P. A. Wolff, Nature (London) 391, 667 (1998).

[5] F. J. García de Abajo, Rev. Mod. Phys. 79, 1267 (2007).

[6] F. J. García-Vidal, L. Martín-Moreno, T. W. Ebbesen, and L. Kuipers, Rev. Mod. Phys. 82, 729 (2010).

[7] R. Gordon, A. G. Brolo, D. Sinton, and K. L. Kavanagh, Laser Photon. Rev. 4, 311 (2010).

[8] R. Ulrich, Infrared Phys. 7, 37 (1967).

[9] R. Ulrich, Infrared Phys. 7, 65 (1967).

[10] B. Blázquez, N. Llombart, D. Cavallo, A. Freni, and A. Neto, IEEE Trans. Antennas Propag. 62, 5077 (2014).

[11] G. G. MacFarlane, J. Inst. Electr. Eng. 93, 703 (1946).

[12] J. Brown, Proc. IEE Part IV: Inst. Monogr. 100, 51 (1953).

[13] J. Brown and W. Jackson, Proc. IEE Part B 102, 11 (1955).
[14] B. A. Munk, Frequency Selective Surfaces: Theory and Design (Wiley, New York, 2000).

[15] M. Al-Joumayly and N. Behdad, IEEE Trans. Antennas Propag. 57, 452 (2009).

[16] B. Gimeno, J. L. Cruz, E. A. Navarro, and V. Such, IEEE Trans. Antennas Propag. 42, 912 (1994).

[17] J. S. Cetnar, J. R. Middendorf, and E. R. Brown, Appl. Phys. Lett. 100, 231912 (2012).

[18] M. A. Hiranandani, A. B. Yakovlev, and A. A. Kishk, IEE Proc.-Microw. Antennas Propag. 153, 487 (2006).

[19] O. Luukkonen, C. Simovski, G. Granet, G. Goussetis, D. Lioubtchenko, A. V. Raisanen, and S. A. Tretyakov, IEEE Trans. Antennas Propag. 56, 1624 (2008).

[20] J. E. Varela and J. Esteban, IEEE Trans. Microwave Theory Tech. 60, 419 (2012).

[21] M. Beruete, M. Sorolla, I. Campillo, J. S. Dolado, L. MartínMoreno, J. Bravo-Abad, and F. J. García-Vidal, Opt. Lett. 29, 2500 (2004). 
[22] M. Beruete, M. Sorolla, I. Campillo, J. S. Dolado, L. MartínMoreno, J. Bravo-Abad, and F. J. García-Vidal, IEEE Trans. Antennas Propag. 53, 1897 (2005).

[23] A. G. Schuchinsky, D. E. Zelenchuk, and A. M. Lerer, J. Opt. A 7, S102 (2005).

[24] A. G. Schuchinsky, D. E. Zelenchuk, A. M. Lerer, and R. Dickie, IEEE Trans. Antennas Propag. 54, 490 (2006).

[25] F. Medina, F. Mesa, and R. Marqués, IEEE Trans. Microwave Theory Tech. 56, 3108 (2008).

[26] N. Marcuvitz, Waveguide Handbook (McGraw-Hill, New York, 1951).

[27] F. Medina, F. Mesa, and D. C. Skigin, IEEE Trans. Microwave Theory Tech. 58, 105 (2010).

[28] A. Khavasi and K. Mehrany, IEEE J. Quantum Electron. 47, 1330 (2011).

[29] E. Yarmoghaddam, G. K. Shirmanesh, A. Khavasi, and K. Mehrany, IEEE Trans. Antennas Propag. 62, 4041 (2014).

[30] T. L. Zinenko, A. I. Nosich, and Y. Okuno, IEEE Trans. Antennas Propag. 46, 1498 (1998).

[31] T. L. Zinenko and A. I. Nosich, IEEE Trans. Antennas Propag. 54, 2088 (1998).

[32] T. L. Zinenko, M. Marciniak, and A. I. Nosich, IEEE J. Sel. Top. Quantum Electron. 19, 9000108 (2013).

[33] C. Molero, R. Rodríguez-Berral, F. Mesa, and F. Medina, IEEE Trans. Microwave Theory Tech. 64, 1195 (2016).

[34] C. Molero, F. Medina, R. Rodríguez-Berral, and F. Mesa, Opt. Express 24, 10265 (2016).

[35] F. Costa, A. Monorchio, and G. Manara, IEEE Antennas Propag. Mag. 54, 35 (2012).

[36] F. Costa, A. Monorchio, and G. Manara, Appl. Comput. Electromagn. Soc. J. 29, 960 (2014).

[37] R. Rodríguez-Berral, F. Medina, F. Mesa, and M. GarcíaVigueras, IEEE Trans. Microwave Theory Tech. 60, 405 (2012).

[38] R. Rodríguez-Berral, C. Molero, F. Medina, and F. Mesa, IEEE Trans. Microwave Theory Tech. 60, 3908 (2012).

[39] R. Rodríguez-Berral, F. Mesa, and F. Medina, IEEE Trans. Antennas Propag. 63, 1969 (2015).

[40] F. Mesa, M. García-Vigueras, F. Medina, R. Rodríguez-Berral, and J. Mosig, IEEE Antennas Wireless Propag. Lett. 14, 135 (2015).

[41] F. Mesa, R. Rodríguez-Berral, M. García-Vigueras, F. Medina, and J. R. Mosig, IEEE Trans. Antennas Propag. 64, 1106 (2016).
[42] J. Brown, Proc. IEE Pt. III 97, 45 (1950).

[43] M. M. Z. Kharadly and W. Jackson, Proc. IEE Pt. III 100, 199 (1953).

[44] D. Cavallo, W. H. Syed, and A. Neto, IEEE Trans. Antennas Propag. 62, 6256 (2014).

[45] D. Cavallo, W. H. Syed, and A. Neto, IEEE Trans. Antennas Propag. 62, 6265 (2014).

[46] S. W. Lee, G. Zarrillo, and C. L. Law, IEEE Trans. Antennas Propag. 30, 904 (1982).

[47] C. S. R. Kaipa, A. B. Yakovlev, F. Medina, F. Mesa, C. A. M. Butler, and A. P. Hibbins, Opt. Express 18, 13309 (2010).

[48] Y. R. Padooru, A. B. Yakovlev, C. S. R. Kaipa, F. Medina, and F. Mesa, Phys. Rev. B 84, 035108 (2011).

[49] Y. R. Padooru, A. B. Yakovlev, C. S. R. Kaipa, G. W. Hanson, F. Medina, and F. Mesa, Phsy. Rev. B 87, 115401 (2013).

[50] M. Kafesaki, I. Tsiapa, N. Katsarakis, T. Koschny, C. M. Soukoulis, and E. N. Economou, Phys. Rev. B 75, 235114 (2007).

[51] M. Beruete, I. Campillo, M. Navarro-Cía, F. Falcone, and M. Sorolla, IEEE Trans. Antennas Propag. 55, 1514 (2007).

[52] B. A. Munk and R. J. Luebbers, IEEE Trans. Antennas Propag. 22, 766 (1974).

[53] B. A. Munk, R. J. Luebbers, and R. D. Fulton, IEEE Trans. Antennas Propag. 22, 804 (1974).

[54] T. Cwik and R. Mittra, IEEE Trans. Antennas Propag. 35, 1397 (1987).

[55] C. Wan and J. A. Encinar, IEEE Trans. Antennas Propag. 43, 1233 (1995).

[56] E. A. Hajlaoui, H. Travelsi, H. Zairi, A. Gharsallah, and H. Baudrand, Microw. Opt. Tech. Lett. 49, 1439 (2007).

[57] C. Molero, R. Rodríguez-Berral, F. Mesa, F. Medina, and A. B. Yakovlev, Phys. Rev. E 93, 013306 (2016).

[58] http://www.ansys.com/, accessed September 2015.

[59] A. P. Hibbins, J. R. Sambles, C. R. Lawrence, and J. R. Brown, Phys. Rev. Lett. 92, 143904 (2004).

[60] D. M. Pozar, Microwave Engineering, 3rd ed. (Wiley, New York, 2005).

[61] J. D. Vacchione and R. Mittra, in Antennas and Propagation Society International Symposium (IEEE, Piscataway, 1990), pp. 1080-1083.

[62] Q. Zhang, T. Guo, B. A. Khan, T. Kodera, and C. Caloz, IEEE Trans. Microwave Theory Tech. 63, 2782 (2015). 